\title{
Joint Power and Admission Control for Multi-pair Massive MIMO AF Relaying System
}

\author{
Yiping Zuo*, Xuesong Liang*, Shi $\mathrm{Jin}^{\dagger}$ and Kai-Kit Wong ${ }^{\ddagger}$ \\ *School of Telecommunication and Information Engineering, \\ Nanjing University of Posts and Telecommunications, Nanjing, China \\ ${ }^{\dagger}$ National Mobile Communications Research Laboratory, Southeast University, Nanjing, China \\ ${ }_{\ddagger}^{\ddagger}$ Department of Electronic and Electrical Engineering, University College London, London, United Kingdom \\ Email:\{1015010328,liangxs\}@ njupt.edu.cn, jinshi@seu.edu.cn, kai-kit.wong@ucl.ac.uk
}

\begin{abstract}
This paper investigates the joint power and admission control problem for a multi-pair massive multiple-input multiple-output (MIMO) amplify-and-forward relaying system. We aim to minimize the total transmit power for users while maximizing the number of supported users by adopting the new linear programming deflation (NLPD) algorithm. Here, the removal strategy of the NLPD algorithm is compared with the deflation based joint user removal and power allocation (DPA) algorithm and the effect of the number of relay's antennas is also analyzed for this massive MIMO relaying system. The MonteCarlo simulation indicates that with thorough removal strategy, the NLPD algorithm can support more users than that of DPA algorithm with almost equal average transmit powers allocated for each user-pair. Moreover, although both two algorithms will accommodate all user-pairs when the number of antennas is sufficiently large, the computational complexity of the NLPD algorithm is at least one order of magnitude lower than the DPA algorithm.
\end{abstract}

\section{INTRODUCTION}

Massive multiple-input multiple-output (MIMO), which is a 5G key technology referred to the idea using few hundreds antennas simultaneously to serve tens of users in the same time-frequency resource [1], has been shown to allow for orders of magnitude improvement in spectral and energy efficiency with using relatively simple (linear) processing. For these benefits, massive MIMO has attracted substantial interest and was extensively studied under different scenarios [1], [2]. Recently, massive MIMO combined with relaying transmission is considered as a strong candidate for the development of future energy-efficient networks and has received increasing attention [3]-[6].

In the literature, some researches have studied ergodic rate analysis [3], [4], precoding analysis [5], [6] and resource allocation [7], [8] in massive relaying MIMO. In particular, the problems of admission control and power allocation are critical for massive MIMO relaying systems when the network contains large number of users [7]-[10]. A long-standing issue associated with power allocation for these scenarios is that the optimal problem often becomes difficult and not all users can be supported simultaneously. Therefore the admission control is necessary to determine some users which have strong interference over the network to be removed in order to make the power allocation problem feasible at all times. In the paper, we study the problem of joint power and admission control for a multi-pair massive MIMO amplify-and-forward (AF) relaying system, which is based on the ergodic signal to interference plus noise ratio (SINR) for each user. Due to the lack of optimal algorithm over this problem, we apply the new linear programming deflation (NLPD) algorithm in [11] to this massive MIMO relaying system, which is compared with the deflation based joint user removal and power allocation (DPA) algorithm in [12]. We show that the NLPD algorithm can support more users with almost equal average transmit powers allocated for each user and with the lower computational complexity.

The remainder of this paper is organized as follows. In Section II, we describe the system model of the multi-pair system with using a one-way massive MIMO AF relay. In Section III, we formulate the problem of joint power and admission control by adopting the NLPD algorithm. The system performance and the computational complexity of the NLPD algorithm are compared with the DPA algorithm and the effect of the number of relay's antennas is analyzed for this massive MIMO relaying system. Our numerical results are provided in Section IV while the conclusion is drawn in Section V.

Notations - Throughout this paper, we use capital boldface letters to denote matrices and small boldface letters to denote column vectors while $(\cdot)^{T},(\cdot)^{H}$ and $\|\cdot\|^{2}$ represent the operations of transposition, conjugate transposition and Euclidean norm, respectively. $E\{\cdot\}$ stands for the expectation of an input random variable.

\section{SySTEM MOdEL}

We consider a multi-pair massive MIMO AF relaying system, where $M$ pairs of source nodes, i.e., $\mathrm{T}_{A_{k}}$, want to communicate with destination nodes, i.e., $\mathrm{T}_{B_{k}}$ for $k=1, \ldots, M$ through an $N$-antenna one-way relay, i.e., $\mathrm{T}_{R}$. We assume that only $K$ user pairs are allowed to communicate with each other at any one time and the NLPD algorithm to select $K$ pairs out of $M$ pairs will be discussed in Section III and we assume $N \gg K$. For this system all the source and destination nodes are equipped with single antenna and we also assumed that the direct links between $\mathrm{T}_{A_{k}}$ and $\mathrm{T}_{B_{k}}$ are broken so that all user pairs need the help of $\mathrm{T}_{R}$ to communicate with each others, whilst the channel between $T_{A_{k}}$ and $T_{R}$ is denoted 
as $\boldsymbol{g}_{k}$ and that between $\mathrm{T}_{B_{k}}$ and $\mathrm{T}_{R}$ is denoted as $\boldsymbol{h}_{k}$ as well, in which $\boldsymbol{g}_{k}$ and $\boldsymbol{h}_{k}$ are statistical independent Rayleigh random vectors with i.i.d. entries, i.e., $\boldsymbol{g}_{k} \sim \mathcal{C N}\left(\mathbf{0}, \sigma_{g_{k}}^{2} \boldsymbol{I}_{N}\right)$ and $\boldsymbol{h}_{k} \sim \mathcal{C N}\left(\mathbf{0}, \sigma_{h_{k}}^{2} \boldsymbol{I}_{N}\right)$ for $k=1, \ldots, M$. Moreover, the transmit power for the relay is represented by $P_{R}$ and that for source terminals are represented by $P_{k}$ for $k=1, \ldots, M$.

The transmission between the user pairs for this multi-pair massive MIMO relaying system take place in two phases. In the first phase, $\mathrm{T}_{A_{k}}$ transmits the information-bearing signals, i.e., $x_{A_{k}}$, for $k=1, \ldots, K$, to $\mathrm{T}_{R}$. Thus, the received signal at $T_{R}$ is expressed as

$$
\boldsymbol{y}_{r}=\boldsymbol{G} \boldsymbol{x}_{A}+\boldsymbol{n}_{R},
$$

where $\boldsymbol{G} \triangleq\left[\boldsymbol{g}_{1}, \ldots, \boldsymbol{g}_{K}\right], \boldsymbol{x}_{A} \triangleq\left[x_{A 1}, \ldots, x_{A K}\right]^{T}$ and $\boldsymbol{n}_{R} \sim$ $\mathcal{C N}\left(\mathbf{0}, \sigma_{R}^{2} \boldsymbol{I}_{N}\right)$, which is the noise vector at $\mathrm{T}_{R}$. In the next phase, after receiving $\boldsymbol{y}_{r}, \mathrm{~T}_{R}$ multiplies it with the amplified coefficient $\rho$, and processes the received signal with the matrix $\boldsymbol{F}$, before it is broadcast to all the users. The transmit signal for $\mathrm{T}_{R}$ is given by

$$
\boldsymbol{y}_{t}=\rho \boldsymbol{F} \boldsymbol{y}_{r} .
$$

It is assumed that $\mathrm{T}_{R}$ has the global CSI, i.e. , $\left\{\boldsymbol{g}_{k}, \boldsymbol{h}_{k}\right\}, \forall k$, and uses this knowledge to construct the processing matrix $\boldsymbol{F}$ while the fixed $\rho$, which constrains the transmit power of $\mathrm{T}_{R}$, is given by

$$
\rho=\sqrt{\frac{P_{R}}{\mathrm{E}\left[\|\mathbf{F G}\|^{2}\right] \boldsymbol{P}+\sigma_{R}^{2} \mathrm{E}\left[\|\mathbf{F}\|^{2}\right]}},
$$

where $\boldsymbol{P}=\operatorname{diag}\left\{P_{1}, \cdots, P_{K}\right\}$, in which $P_{k}$ is the transmit power of $x_{A_{k}}$, for $k=1, \cdots, K$. In this paper, we assume that $\mathrm{ZF}$ criterion is used at relay with the processing matrix $\boldsymbol{F}$ expressed as

$$
\boldsymbol{F}=\boldsymbol{H}\left(\boldsymbol{H}^{H} \boldsymbol{H}\right)^{-1}\left(\boldsymbol{G}^{H} \boldsymbol{G}\right)^{-1} \boldsymbol{G}^{H},
$$

where $\boldsymbol{H} \triangleq\left[\boldsymbol{h}_{1}, \ldots, \boldsymbol{h}_{K}\right]$. Finally, $\boldsymbol{T}_{B_{k}}$ receives

$$
z_{B_{k}}=\boldsymbol{h}_{k}^{H} \boldsymbol{y}_{t}+n_{B_{k}}
$$

with $n_{B_{k}} \sim \mathcal{C N}\left(0, \sigma_{B_{k}}^{2}\right)$, which is the noise vector at $T_{B_{k}}$, for $k=1, \ldots, K$. In the sequel, the received signal is given by

$$
z_{B_{k}}=\underbrace{\rho x_{A_{k}}}_{\text {Signal }}+\underbrace{\rho\left[\left(\boldsymbol{G}^{H} \boldsymbol{G}\right)^{-1} \boldsymbol{G}^{H}\right]_{k} \boldsymbol{n}_{R}+n_{B_{k}}}_{\text {Interference and Noise }},
$$

for $k=1, \ldots, K$. Then ergodic SINR for $\mathrm{T}_{B_{k}}$ is expressed as

$$
\gamma_{B_{k}}^{Z}=\mathrm{E}\left[\frac{P_{k}}{\sigma_{R}^{2}\left[\left(\boldsymbol{G}^{H} \boldsymbol{G}\right)^{-1}\right]_{k, k}+\sigma_{B_{k}}^{2} / \rho^{2}}\right] \text {, for } k=1, \ldots, K
$$

Here we aim to use $\gamma_{B_{k}}^{Z}$ to evaluate the actual SINR of $T_{B_{k}}$, however the calculation for $\gamma_{B_{k}}^{Z}$ is challenging and infeasible to get closed-form expression of that. Then we use a lower bound for $\gamma_{B_{k}}^{Z}$ (which is proved to be extremely tight) in [4] as following

$$
\gamma_{B_{k}}=\frac{(N-K) P_{k}}{\frac{\sigma_{R}^{2}}{\sigma_{g_{k}}^{2}}+\frac{\sigma_{B_{k}}^{2}}{P_{R}} \sum_{j=1}^{K} \frac{P_{j}}{\sigma_{h_{j}}^{2}}+\frac{\sigma_{R}^{2} \sigma_{B_{k}}^{2}}{(N-K) P_{R}} \sum_{j=1}^{K} \frac{1}{\sigma_{g_{j}}^{2} \sigma_{h_{j}}^{2}}},
$$

for $k=1, \ldots, K$.

\section{OPTIMAL JOINT POWER AND ADMISSION CONTROL PROBLEM}

Now we have the closed-form of lower bound for $\gamma_{B_{k}}^{Z}$ and we proceed to the joint power and admission control problem for this massive MIMO relaying system, which can be mathematically formulated as a two-stage optimization problem in [11], containing $M 1$ and $M 2$. The problem $M 1$ in the first stage is the admission control problem, which aims to maximize the number of the supported users while satisfying the stringent SINR requirement of each user and the transmit power constraints. Specifically, the admission control problem can be formulated as

$$
M 1:\left\{\begin{array}{l}
\max _{p, S}|S| \\
\text { s.t. } \gamma_{B_{k}} \geq \bar{\gamma}_{k}, \quad k \in S \subseteq \mathcal{M} \\
\boldsymbol{P}^{\min } \leq \boldsymbol{P} \leq \boldsymbol{P}^{\max }
\end{array}\right.
$$

where $\mathcal{M}$ represents the index set for all user pairs, i.e., $\mathcal{M}=\{1,2, \cdots, M\}$ and $\mathrm{S}$ represents the index subset for the selected user pairs, i.e., $S=\{1,2, \cdots, K\} .|S|$ denotes the cardinality of the set $\mathrm{S}$ and $\bar{\gamma}_{k}$ is the $k$-th user's SINR threshold. We denote the power allocation vector by $\boldsymbol{P}=$ $\left(P_{1}, \cdots, P_{K}\right)^{T}$ and the transmit power constraints by $\boldsymbol{P}^{\mathrm{min}}=$ $\left(P_{1}^{\min }, \cdots, P_{K}^{\min }\right)^{T}$ and $\boldsymbol{P}^{\max }=\left(P_{1}^{\max }, \cdots, P_{K}^{\max }\right)^{T}$.

The problem $M 2$ in the second stage is the power allocation problem, we purport to minimize the total transmit power for users under the same constraints with the above problem (9). Therefore the power allocation problem can be expressed as

$$
M 2:\left\{\begin{array}{l}
\min _{\left\{P_{k}\right\}_{k} \in S_{0}} \sum_{k \in S_{0}} P_{k} \\
\text { s.t. } \gamma_{B_{k}} \geq \bar{\gamma}_{k}, k \in S_{0} \\
\quad P_{k}^{\min } \leq P_{k} \leq P_{k}^{\max }, k \in S_{0}
\end{array}\right.
$$

We use $S_{0}$ to denote the maximum admitted user set for the problem (9). Notice that maximum admitted user set $S_{0}$ might not be unique. For a given admissible set $S_{0}$, the power allocation problem (10) is feasible, and the problem can be efficiently solved in a distributed fashion [13]. However, admission control problem (9) of finding the maximum admissible set S is NP-hard. The complexity result motivates us to apply the NLPD algorithm in [11] for this massive MIMO relaying system to solve the joint power and admission control optimization problem.

Without loss of generality, we assume $\sigma_{R}^{2}=\sigma_{B_{k}}^{2}=c$, and substitute (8) into the SINR constraints for the problem $M 1$ 
and $M 2$, and recast them as follows

$$
\frac{(N-K) P_{k}}{\underbrace{c \tilde{g}_{k}}_{P 1}+\underbrace{\frac{c}{P_{R}} \sum_{j=1}^{K} P_{j} \tilde{h}_{j}}_{P 2}+\underbrace{\frac{c^{2}}{(N-K) P_{R}} \sum_{j=1}^{K} \tilde{g}_{j} \tilde{h}_{j}}_{P 3}} \geq \bar{\gamma}_{k},
$$

where $\tilde{g}_{k}=\frac{1}{\sigma_{g_{k}}^{2}}$ and $\tilde{h}_{k}=\frac{1}{\sigma_{h_{k}}^{2}}$, for $k=1, \ldots, K$. The above inequality can be further simplified, specifically, noting that the SINR threshold $\bar{\gamma}_{k}>1$ is always satisfied in the wireless communications, we obtained the Lemma 1 as following.

Lemma 1: Assuming $\bar{\gamma}_{k}>1$, we obtained $P 2 \gg P 3$ for $N-K \gg 1$, which indicates that $P 3$ can be neglected when the number of relay's antennas is sufficiently large. Therefore the expression (11) can be further simplified as

$$
\frac{(N-K) P_{k}}{\underbrace{c \tilde{g}_{k}}_{P 1}+\underbrace{\frac{c}{P_{R}} \sum_{j=1}^{K} P_{j} \tilde{h}_{j}}_{P 2}} \geq \bar{\gamma}_{k}, \text { for } k=1, \ldots, K .
$$

The proof of Lemma 1 is provided in Appendix A.

Our aim is to minimize the total transmit power for users while maximizing the number of satisfied users, we applied the NLPD algorithm referred in [11] and Lemma 1 in this massive MIMO relaying system to merge two problems, (9) and (10) into the following linear program

$$
\left\{\begin{array}{cl}
\min _{\mathbf{q}} & \mathbf{e}^{T}(\mathbf{c}-\mathbf{A q})+\alpha\left(\mathbf{p}^{\max }\right)^{T} \mathbf{q} \\
\text { s.t. } & \mathbf{c}-\mathbf{A q} \geq \mathbf{0} \\
& \mathbf{P}^{\min } / \mathbf{P}^{\max } \leq \mathbf{q} \leq \mathbf{e}
\end{array}\right.
$$

where e is $M \times 1$ identity matrix, $\mathbf{q}=\left(q_{1}, q_{2}, \ldots, q_{M}\right)^{T}$ with $q_{k}=\frac{P_{k}}{P_{k}^{\max }}$ and $\mathbf{c}=\left(c_{1}, c_{2}, \ldots, c_{M}\right)^{T}$ with $c_{k}=\frac{\gamma_{k}^{\prime} \eta_{k}}{a P_{k}^{\max }}, \forall k \in$ $\mathcal{M}$, and the entries of the channel matrix $\mathbf{A} \in \mathcal{R}^{M^{k} \times M}$ is

$$
a_{k j}= \begin{cases}1, & \text { if } k=j \\ -\frac{\gamma_{k}^{\prime} g_{k j} P_{j}^{\max }}{a P_{k}^{\max }}, & \text { if } k \neq j,\end{cases}
$$

where $a=N-K, \eta_{k}=c \tilde{g}_{k}, g_{k j}=\frac{c \tilde{h}_{j}}{P_{R}}$ and $\gamma_{k}^{\prime}=\frac{\bar{\gamma}_{k}}{1-\frac{g_{k k} \gamma_{k}}{a}}$ for $k, j=1, \ldots, M$, and the parameter $\alpha$ satisfying

$$
\alpha= \begin{cases}c_{1} \alpha_{1}, & \text { if } \rho(\mathbf{I}-\mathbf{A}) \geq 1 \\ c_{2} \min \left\{\alpha_{1}, \alpha_{2}\right\} & \text { if } \rho(\mathbf{I}-\mathbf{A})<1\end{cases}
$$

where $\alpha_{1}=\frac{1}{\left(\mathbf{P}^{\max }\right)^{T} \mathbf{e}}, \alpha_{2}=\frac{1}{\max \left\{\left(\mathbf{A}^{T}\right)^{-1} \mathbf{P}^{\max }\right\}}, 0<c_{1} \leq$ $c_{2}<1$ and $c_{1}, c_{2}$ are two constants.

We know that $(\mathbf{c}-\mathbf{A q})_{k}$ denotes the excess transmit power of the $k$-th user. Therefore, the linear program (13) actually minimizes a weighted sum of the total excess transmit power $\mathbf{e}^{T}(\mathbf{c}-\mathbf{A q})$ and the total real transmit power $\left(\mathbf{p}^{\max }\right)^{T} \mathbf{q}$. The proof of (13) can be found in Appendix B. The basic idea of the above mentioned NLPD algorithm in the preprocessing is to solve the problem (13) and check whether all users can be admitted or not. In this paper, the same argument as in the removal strategy used in [11] can be applied to recast
(9) and (10) to an equivalent optimization problem (13). The description of the NLPD algorithm for this massive MIMO relaying system is given as follows.

\section{A NLPD Algorithm for This Massive MIMO Relaying System}

Step 1 Initialization: Input data $\left(\boldsymbol{A}, \boldsymbol{c}, \mathbf{P}^{\min }, \mathbf{P}^{\max }\right)$.

Step 2 Preprocessing: Remove user $k_{0}$ iteratively according to $k_{0}=\arg \max _{k \in \mathcal{M}}\left\{\sum_{j \neq k}\left|a_{k j}\right|+\sum_{j \neq k}\left|a_{j k}\right|+c_{k}\right\}$, until necessary condition $\max \left\{\mathbf{A}^{T} \mathbf{e}, 0\right\}^{T} \mathbf{e}-(\max \{-$ $\left.\left.\mathbf{A}^{T} \mathbf{e}, 0\right\}+\mathbf{e}\right)^{T} \mathbf{c} \geq 0$ holds true.

Step 3 Power Allocation: Solve the liner programming problem (13) to check whether all users are supported: if yes, go to end; else go to Step 4.

Step 4 Admission Control: Drop user $k_{0}$ according to $k_{0}=$ $\arg \max _{k \in \mathcal{M}}\left\{\sum_{j \neq k}\left|a_{k j}\right| q_{k}^{e}+\sum_{j \neq k}\left|a_{j k}\right| q_{j}^{e}\right\}$ with $q_{k}^{e}=$ $(\mathbf{c}-\mathbf{A q})_{k}$ and set $\mathcal{M}=\mathcal{M} / k_{0}$, and go to Step 3 .

Next, we will study the removal strategy of the NLPD algorithm compared with the DPA algorithm and analyze the effect of the number of relay's antennas for this massive MIMO relaying system. In the admission control process, the removal strategy of the NLPD algorithm to drop the user $k_{0}$ is expressed as

$$
k_{0}=\arg \max _{k \in \mathcal{M}}\{S 1+S 2\},
$$

where $S 1=\sum_{j \neq k}\left|a_{k j}\right| q_{k}^{e}$ and $S 2=\sum_{j \neq k}\left|a_{j k}\right| q_{j}^{e}$. Firstly, we study the removal strategy of the NLPD algorithm compared with the DPA algorithm. The basic idea of the removal strategy of the NLPD algorithm is elaborated as follows. Note that the $q_{k}^{e}$ in (16) indicates the excess transmission power needed by $\mathrm{T}_{A_{k}}$ to fulfill its desired SINR target, with assuming all the other users' transmit powers unchanged. Therefore extra interferences are caused by $q_{k}^{e}$ from user- $k$ to the other transmitters. Let us denote $S 1$ as the sum of excess interference from user- $k$ to all other transmitters and denote $S 2$ as that from all other transmitters to user $k$. Therefore these two issues come into being the removal strategy (16). Different from the above removal strategy, the DPA algorithm in [12] is given by

$$
k_{0}=\arg \max _{k \in \mathcal{M}}\left\{\bar{\gamma}_{k}-\gamma_{B_{k}}\right\} .
$$

The basic idea of this algorithm is distributed iteration. For a given user set, the user with the largest gap to its SINR threshold as in (17) is removed from the set of admissible users until the admissible set meets the required SINR thresholds. Comparing (16) and (17), we can see that the removal strategy (16) is more thoughtful, not by the gap to its SINR threshold only as in (17) but by the compound interference measured by the weighted excess transmit power. Therefore the possibility of removing users of the NLPD algorithm by accident is overwhelming smaller than the DPA algorithm.

Secondly, we will analyze the effect of $N$ on this massive MIMO relaying system. After substituting the expressions of 
$a_{k j}$ and $q_{k}^{e}$ into (16), we can obtain above removal strategy $k_{0}$ in original form. The expression related to $N$ is given by

$$
\begin{aligned}
k_{0}=\arg \max _{k \in \mathcal{K}}\left\{\frac{1}{N-K} \sum_{j \neq k}(\underbrace{X_{k j}\left(\frac{\bar{\gamma}_{k}}{N-K}+Y_{k j}\right)}_{F 1}\right. \\
+\underbrace{X_{j k}\left(\frac{\bar{\gamma}_{j}}{N-K}+Y_{j k}\right)}_{F 2})\}
\end{aligned}
$$

where $X_{k j}=\bar{\gamma}_{k} g_{k j} P_{j}^{\max }\left(\eta_{k}+\sum_{j \neq k}^{K} g_{k j} P_{j}\right) /\left(P_{k}^{\max }\right)^{2}$, $Y_{k j}=P_{k} / c \tilde{g}_{k}+\sum_{j=1}^{K} g_{k j} \tilde{h}_{j}$ and $X_{k j}, Y_{k j}$ are two coefficients independent of $N$. In the removal strategy of NLPD algorithm, the user $k_{0}$ in (18) is dropped when $\gamma_{B_{k_{0}}}<\bar{\gamma}_{k}$. It is shown from (18) that the compound interference which measured by $S 1+S 2$ is at least inversely proportional to $(N-K)$. Therefore with the increase of $N$, the $k_{0}$-th user's SINR will become larger and nearer to its SINR threshold. In a word, more user-pairs will be supported simultaneously when $N$ increases, and all the user-pairs will be supported simultaneously if $N$ is large enough. Besides, when $N$ increases, the impact of the SINR threshold $\bar{\gamma}_{k}$ in the bracket of $F 1$ on $S 1+S 2$ is weakened, and so does $\bar{\gamma}_{j}$ in the bracket of $F 2$. It imply that the influence of the SINR thresholds disparity among all users is weakened by the increase of $N$, while $Y_{k j}$ which is approximately the weighted $\gamma_{B_{k}}$ will play a more important role in the compound interference.

\section{Numerical Results}

In this section, we provide some numerical examples in order to show the benefits of the NLPD algorithm for this massive MIMO relaying system. All figures are averaged over 200 Monte-Carlo runs. Without loss of generality, we assume that the transmitters and receivers are generated randomly and uniformly in a disk around the one-way relay and radius $400 \mathrm{~m}$, excluding a radius of $10 \mathrm{~m}$. The angle between the location of each pair of transmitter and receiver is larger than 90 degrees and less than 270 degrees, using the relay as vertex. Each user's SINR target is set as $\bar{\gamma}_{k}=50 d B(\forall k \in \mathcal{M})$ and the noise power is set as $\sigma_{R}^{2}=\sigma_{B_{k}}^{2}=-90 \mathrm{dBm}(\forall k \in \mathcal{M})$. We also assume that the power of relay is $P_{R}=20 \mathrm{~W}$ and the transmit power constraints of the user $k$ are $P_{k}^{\min }=0.001 \mathrm{~W}$ and $P_{k}^{\max }=20 W(\forall k \in \mathcal{M})$, respectively. The parameters $c_{1}$ and $c_{2}$ in (15) are set as 0.1 and 0.999 , respectively, and the number of relay's antennas is set to $N=200$ or 300 . The system performance is evaluated in terms of average number of supported users, transmit power allocated for each user, and execution time. The performance of the NLPD algorithm in this massive MIMO relaying systems as shown as follows.

In Fig. 1 we compare the average number of supported users between the NLPD and DPA algorithms for $N=200$

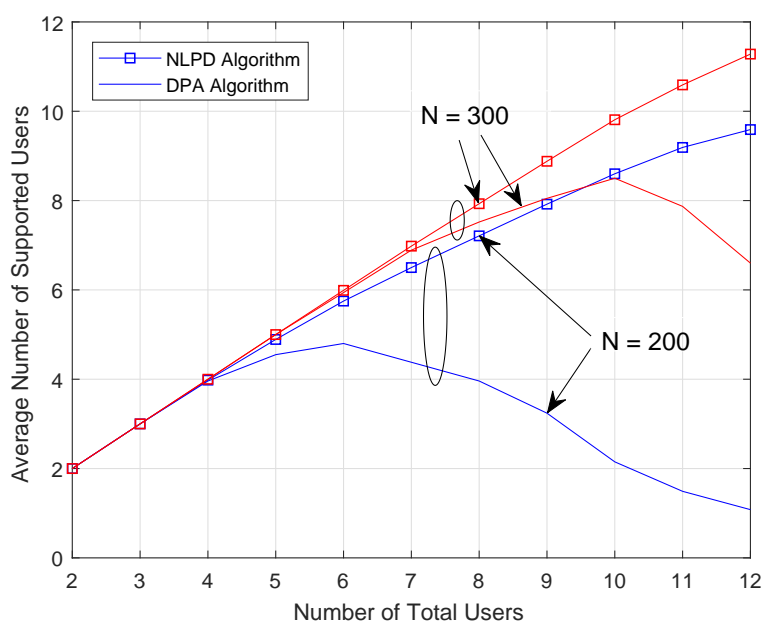

Fig. 1: Average number of supported users versus the number of total users.

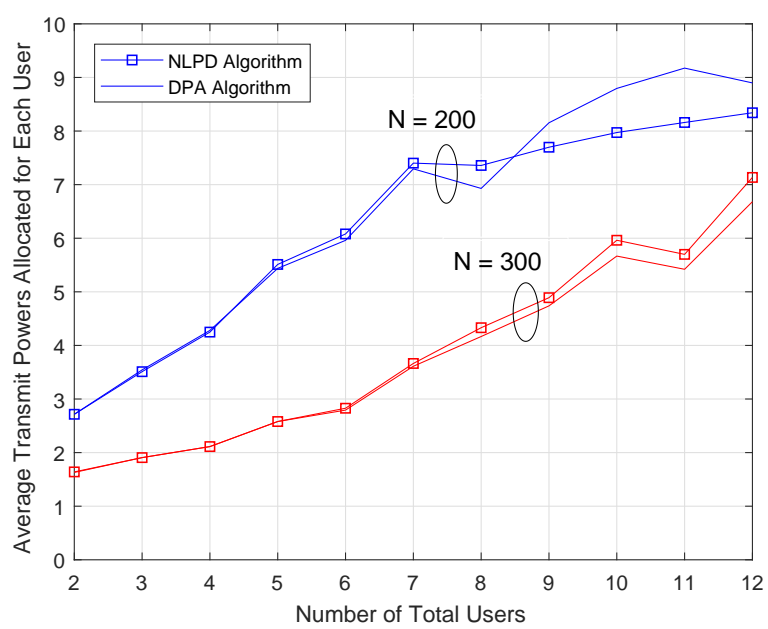

Fig. 2: Average transmit powers allocated for each user versus the number of total users.

TABLE I: Average execution time comparison $(N=200)$

\begin{tabular}{|c|c|c|}
\hline \multirow{2}{*}{ Number of total users } & \multicolumn{2}{|c|}{ Total time / Time for each user (second) } \\
\cline { 2 - 3 } & NLPD & DPA \\
\hline 2 & $39.80 / 19.90$ & $307.20 / 153.60$ \\
\hline 4 & $41.73 / 10.43$ & $747.88 / 186.97$ \\
\hline 6 & $48.95 / 8.16$ & $1728.95 / 288.16$ \\
\hline 8 & $67.94 / 8.49$ & $4232.06 / 529.01$ \\
\hline 10 & $95.19 / 9.52$ & $8420.01 / 842.00$ \\
\hline 12 & $118.65 / 9.89$ & $13354.25 / 1112.85$ \\
\hline
\end{tabular}

or $N=300$. Results show that these two algorithms can both support all users for a small total users number, e.g. , $M \leq 4$, and the number of admitted users for two algorithms both increase with the increase of $N$. However, the increase of the DPA algorithm is significantly lower than the NLPD algorithm. Moreover, although the performance of the DPA algorithm is greatly improved when $N$ increases, the number of admitted users will drop dramatically while $M$ exceeds a 
certain threshold. By contrast, the number of admitted users of the NLPD algorithm increases near-linearly with the increase of $M$ and the performance of the NLPD algorithm is much more close to full user scheduling with the increase of $N$.

Fig. 2 demonstrates the average transmit powers allocated for each user by both two algorithms. Results in Fig. 2 illustrate that the average transmit powers allocated for each user are nearly equal for these two algorithms, and this conclusion still holds when $N$ increases. Combined with Fig. 1, it is inferred that the NLPD algorithm can schedule more users than the DPA algorithm with almost equal average transmit powers for the supported users. For instance, when $M=12$ and $N=200$, the average number of supported users of the NLPD algorithm is 8.51 times as large as that of the DPA algorithm, while the average transmit powers allocated for each user is even 0.56 less than the DPA algorithm. It is shown that the NLPD algorithm greatly improves the number of accommodated users with hardly increasing interference of system than the DPA algorithm.

Finally, the average execution time is compared between the NLPD and DPA algorithms for $N=200$. As observed from Table. I, the average execution time for each user of the NLPD algorithm is basically stable and is slightly enhanced when $M>4$. However, it is relatively large for $M=2$ and $M=4$ because of some fixed time-sharing operating bearing, such as the initializing input data and preprocessing. Meanwhile, the amount of computation of the DPA algorithm is nearly exponential growth with the increase of $M$. Therefore, the NLPD algorithm has a significant advantage in the computational complexity when $M$ increases. For example, when $M=2$, the average execution time of the DPA algorithm is about 7.72 times as large as that of the NLPD algorithm, and the multiple factor is added up to 112.55 for $M=12$.

\section{CONCLUSION}

In this paper, we studied the joint power and admission control problem and analyzed the system performance and the computational complexity of the NLPD algorithm compared with the DPA algorithm for a multi-pair massive MIMO AF relaying system. It is shown that the NLPD algorithm can support more users with almost equal average transmit powers allocated for each user and achieve significantly less execution time than the DPA algorithm. Moreover, simulation results reveal that more user-pairs can be supported simultaneously by this massive MIMO relaying system when the number of relay's antennas increases. Meanwhile, it is also shown that the increase of the the number of relay's antennas diminishes the impact of different SINR thresholds of user-pairs.

\section{APPENDIX A}

PROOF OF LEMMA 1

From the inequality (11), we can easily obtain the expression as follows

$$
(N-K) P_{k}>\bar{\gamma}_{k} \max \{P 1, P 2, P 3\}, \text { for } k=1, \ldots, K .
$$

As $\bar{\gamma}_{k}>1$, we recast (19) into following inequality

$$
(N-K) P_{k}>c \bar{\gamma}_{k} \tilde{g}_{k}>c \tilde{g}_{k} .
$$

Then we multiply (20) by $c \tilde{h}_{k} / P_{R}$ and have summation with respect to $k$ on the two sides together, hence we obtained

$$
\frac{c}{P_{R}} \sum_{k=1}^{K} P_{k} \tilde{h}_{k}>\frac{c^{2}}{(N-K) P_{R}} \sum_{k=1}^{K} \tilde{g}_{k} \tilde{h}_{k},
$$

which indicates that $P 2>P 3$ for any value of $N-K$. We note that $P 2$ is a constant value with respect to $N-K$ whilst $P 3$ is linearly decreased with $N-K$, then we obtained $P 2 \gg P 3$ when $N-K \gg 1$.

\section{APPENDIX B}

DERIVATION DETAILS OF (13)

To begin with, we recast the problem (9) and (10) as follows

$$
M 1: \quad\left\{\begin{array}{l}
\max _{P, S}|S| \\
\text { s.t. } \gamma_{B_{k}}^{\prime} \geq \gamma_{k}^{\prime}, k \in S \subseteq \mathcal{M} \\
\boldsymbol{P}^{\text {min }} \leq \boldsymbol{P} \leq \boldsymbol{P}^{\max }
\end{array}\right.
$$

and

$$
M 2:\left\{\begin{array}{l}
\min _{\left\{P_{k}\right\}_{k} \in S_{0}} \sum_{k \in S_{0}} P_{k} \\
\text { s.t. } \gamma_{B_{k}}^{\prime} \geq \gamma_{k}^{\prime}, k \in S_{0} \\
\quad P_{k}^{\min } \leq P_{k} \leq P_{k}^{\max }, k \in S_{0},
\end{array}\right.
$$

with $\gamma_{B_{k}}^{\prime}=\frac{a P_{k}}{\eta_{k}+\sum_{j \neq k, j=1}^{K} g_{k_{j}} P_{j}}$ and $\gamma_{k}^{\prime}=\frac{\bar{\gamma}_{k}}{1-\frac{g_{k k} \gamma_{k}}{a}}$ for $k, j=$ $1, \ldots, K$. The problem (22) and (23) are the standard formulas as defined in [11], which can be merged into a linear program (13) by using the NLPD algorithm for this massive MIMO relaying system.

To facilitate the development of joint power and admission control problem, we normalized the channel parameters to obtain an equivalent normalized channel. In particular, let us use $\mathbf{q}=\left(q_{1}, q_{2}, \ldots, q_{M}\right)^{T}$ with $q_{k}=\frac{P_{k}}{P_{k}^{\max }}$ to denote the normalized power allocation vector, then $\gamma_{B_{k}}^{\prime} \geq \gamma_{k}^{\prime}$ had been normalized as follows

$$
\frac{q_{k}}{\frac{\gamma_{k}^{\prime} \eta_{k}}{a P_{k}^{\max }}+\sum_{j \neq k, j=1}^{K} \frac{\gamma_{k}^{\prime} g_{k j} P_{j}^{\max }}{a P_{k}^{\max }} q_{j}} \geq 1 .
$$

We used $\mathbf{c}=\left(c_{1}, c_{2}, \ldots, c_{M}\right)^{T}$ with $c_{k}=\frac{\gamma_{k}^{\prime} \eta_{k}}{a P_{k}^{\max }}$, for $k, j=$ $1, \ldots, M$, to indicate the normalized noise vector. We also defined a normalized channel matrix $\mathbf{A} \in \mathcal{R}^{M \times M}$ by

$$
a_{k j}= \begin{cases}1, & \text { if } k=j \\ -\frac{\gamma_{k}^{\prime} g_{k j} P_{j}^{\max }}{a P_{k}^{\max }}, & \text { if } k \neq j\end{cases}
$$

where $a_{k j}$ indicates the $(k, j)$-th entry of $\mathbf{A}$, and $\left|a_{k j}\right|$ is the normalized channel gain from the transmitter of user $j$ to the receiver of user $k$, for $k, j=1, \ldots, M$. Hence the linear program (13) is obtained. 


\section{REFERENCES}

[1] E. G. Larsson, O. Edfors, F. Tufvesson, and T. L. Marzetta, "Massive MIMO for next generation wireless systems," IEEE Commun. Mag., vol. 52, no. 2, pp. 186-195, Feb. 2014.

[2] E. Bjornson, E. G. Larsson, and T. L. Marzetta, "Massive MIMO: ten myths and one critical question," IEEE Commun. Mag., vol. 54, no. 2, pp. 114-123, Feb. 2016.

[3] S. Jin, M. R. McKay, C. Zhong, and K. K. Wong, "Ergodic capacity analysis of amplify-and-forward MIMO dual-hop systems," IEEE Trans. Inform. Theory, vol. 56, no. 5, pp. 2204-2224, May 2010.

[4] S. Jin, X. Liang, K. K. Wong, X. Gao, and Q. Zhu, "Ergodic rate analysis for multipair massive MIMO two-way relay networks," IEEE Trans. Wireless Commun., vol. 14, no. 3, pp. 1480-1491, Mar. 2015.

[5] J. Liu, W. Xu, S. Jin, and X. Dong, "RF-chain constrained multi-pair massive MIMO relaying using hybrid precoding and detection," in Proc. IEEE Wireless Communications and Networking Conference (WCNC), Apr. 2016, pp. 1-6.

[6] H. M. Wang, K. W. Huang, Q. Yang, and Z. Han, "Joint source-relay secure precoding for MIMO relay networks with direct links," IEEE Trans. Commun., vol. PP, no. 99, pp. 1-13, 2017.

[7] H. Gao, T. Lv, X. Su, H. Yang, and J. M. Cioffi, "Energy-efficient resource allocation for massive MIMO amplify-and-forward relay systems," IEEE Access, vol. 4, pp. 2771-2787, 2016.

[8] H. Liu, H. Gao, S. Yang, and T. Lv, "Low-complexity downlink user selection for massive MIMO systems," IEEE Systems J., vol. PP, no. 99, pp. 1-12, 2017.

[9] Q. Zhang, S. Jin, M. McKay, D. Morales-Jimenez, and H. Zhu, "Power allocation schemes for multicell massive MIMO systems," IEEE Trans. Wireless Commun., vol. 14, no. 11, pp. 5941-5955, Nov. 2015.

[10] Y. Dai and X. Dong, "Power allocation for multi-pair massive MIMO two-way af relaying with linear processing," IEEE Trans. Wireless Commun., vol. 15, no. 9, pp. 5932-5946, Sep. 2016.

[11] Y. F. Liu, Y. H. Dai, and Z. Q. Luo, "Joint power and admission control via linear programming deflation," IEEE Trans. Signal Process., vol. 61, no. 6, pp. 1327-1338, Mar. 2013.

[12] J. Yan, J. Li, and L. Zhao, "Joint user scheduling and power allocation with quality of service guarantees in downlink distributed antennas system," IET Commun., vol. 8, no. 3, pp. 299-307, Feb. 2014.

[13] E. Karamad and R. S. Adve, "On the stability of distributed power control algorithms under imperfect estimation of channel and interference," IEEE Trans. Commun., vol. PP, no. 99, pp. 1-1, 2017. 